\title{
Trombosis venosas en el estado grávido-puerperal
}

\section{Doctor Jesús Alberto Gómez Palacino (:)}

Con este título hemos querido reunir en un solo concepto las entidades venosas antiguamente consideradas de naturaleza séptica y por consiguiente con fenómenos fisiopatológicos de orden inflamatorio. Una revisión de este tema nos permitirá aclarar algunas ideas hoy revaluadas de acuerdo con un conocimiento más profundo del problema.

Hasta hace relativo corto tiempo el factor etiológico de estas complicaciones se hacía recaer en la infección; se atribuía a las noxas inflamatorias un papel predominante. De acuerdo con este criterio se hablaba de flebitis, tromboflebitis y flebotrombosis, haciendo inclusive una diferenciación anatomo-clínica de cađa una de estas entidades. Esta nomenclatura, en lugar de aclarar el problema, conllevaba una idea confusa a su estudio. Estableciendo tal diferencia se pensó en entidades diferentes cuando la realidad es que se trata de distintos estados evolutivos de una misma entidad: la trombosis venosa.

De acuerdo con las modernas investigaciones de la escuela francesa, un fenómeno caracteriza a estos estados y constituye su fisiopatología: la hipercoagulabilidad del plasma. No está bien clara la causa íntima de esta hipercoagulabilidad plasmática, pero entre sus factores determinantes parece jugar gran papel el aspecto endocrino. Como causas desencadenantes se invocan: las cardiopatías, la obesidad, la desnutrición, la infección, las hemorragias, la deshidratación y los traumatismos quirúrgicos u obstétricos. Desde luego es necesario tener en cuenta que siendo un fenómeno bioquímico intravascular, se vea favorecido por las lesiones o modificaciones de la anatomofisiología de su continente, o sea de las venas.

Desde el punto de vista patogénico es necesario poner de presente tres factores que intervienen en el mecanismo de las trombosis venosas: el factor bioquímico, el factor hemodinámico y el factor parietal (1).

(*) Asistente de Cátedra. Instituto de Protección Materna e Infantil "Concepción Villaveces de Acosta". Bogotá. Colombia. 
Factor bioquímico: caracteriza a las trombosis y es la manifestación de un aumento de los elementos químicos que intervienen en los procesos de la coagulación sanguínea: protrombina, tromboplastina, etc., y una disminución de las substancias anticoagulantes, principalmente de la heparina.

Factor hemodinámico: se manifiesta por un retardo en la circulación venosa de retorno que se ve favorecido por los estados de reposo prolongado y las enfermedades cardiacas que disminuyen el impulso sanguíneo inicial. A menor velocidad de circulación sanguínea en el territorio venoso, mayor posibilidad de formación de trombos.

Factor parietal: la lesión anatómica y funcional de la pared venosa constituye un terreno favorable en la producción de las trombosis. De ahí que estos trastornos de la coagulación sanguirea sean más frecuentes en pacientes varicosas en quienes el tono de la muscular venosa está disminuído, la circulación será más lenta y las posibilidades de formación de coágulos mayor. Por otra parte, es necesario recordar que la nutrición de la pared venosa se hace por dos mecanismos: el primero, por la sangre que circula en contacto directo, y el segundo por intermedio de los vasa vasorum. De ahí que los estados prolongados de anemia conduzcan a la lesión del endotelio venoso por un mecanismo de anoxia celular.

De lo dicho anteriormente se puede deducir la razón por la cual las trombosis venosas son mucho más frecuentes en los miembros inferiores y en los vasos de la pelvis que en otras partes del organismo, en donde son relativamente raras.

Ordinariamente el proceso patológico de la coagulación intravascular se inicia en las venas musculares de las piernas y con menor frecuencia en las venas de la planta del pie, y puede adoptar dos modalidades clínicas, según que el coágulo se adhiera y obstruya la circulación o que flote sin producir obstrucción; en ei segundo caso puede suceder inclusive que no se encuentre al estudio anatomopatológico sitio de implantación del coágulo sobre la pared venosa.

En los casos en que ocurre obstrucción venosa tal fenómeno se acompaña de grandes alteraciones que confieren caracteres inflamatorios a la lesión, porque al trastorno endotelial grave responde todo el paquete vásculo-nervioso con espasmo de la arteria correspondiente que lleva a la isquemia e inclusive a la gangrena de los miembros. Habrá además edema, que se explica por el aumento de la presión venosa y el aumento de la permeabilidad ca- 
pilar. Todas estas manifestaciones explican el intenso dolor que acompaña a estos trastornos.

Cuando el trombo no obstruye la vena es posible que no haya manifestaciones locales y tan solo sea apreciable un aumento de ia frecuencia del pulso en discordancia con la temperatura; de ahí que potencialmente esta forma clínica sea mucho más peligrosa.

A manera de conclusión podemos decir que la trombosis venosa se manifiesta por alteraciones generales y locales. Las primeras son: leves ascensos de la temperatura; aumento de la frecuencia del pulso, siempre en discordancia con la temperatura; malestar general, y sensación angustiosa con situación psicológica de pesimismo. Las manifestaciones locales dependen de que haya o no obstrucción venosa. En el primer caso se presenta: dolor en el miembro afectado y particularmente en la pantorrilla (signo de Homas) y a lo largo del trayecto venoso (safena, polplítea, femoral, iliaca externa), hipertermia local, edema apreciable, impotencia funcional y cianosis de las uñas. En los casos avanzados es frecuente el espasmo arterial secundario, que provoca isquemia con palidez del miembro enfermo y que inclusive puede llegar a la gangrena.

Cuando el proceso compromete las venas pélvicas, aparece meteorismo intestinal con alteraciones de la micción y la defecación, contractura del músculo psoas si la alteración alcanza la vena cava y lo más frecuente: edema de los genitales externos.

Si el coágulo permanece más o menos libre y flota en la luz de la vena, es frecuente que no haya manifestaciones locales, lo cual confiere particular gravedad al fenómeno, ya que sus manifestaciones clínicas tardias y a distancia generalmente son muy serias, pues dependen del desprendimiento del coágulo con la posibilidad inminente de tromboembolias de grave pronóstico.

Desde el punto de vista obstétrico es necesario tener presente cuue estos estados de hipercoagulabilidad plasmática se pueden presentar durante el estado de gravidez o, lo qué es más frecuente, durante el post-parto o en el puerperio tardío. Por fortuna las trombosis venosas son de rara ocurrencia on el curso del embarazo. La estadística americana reportada (2) reúne 135 casos, y la del Fiiadelfia Lying-in Hospital registra 9 casos en 20 años.

Lamentablemente la trombosis durante $\in 1$ período puerperal sigue siendo frecuente y de ahí la importancia que implica el manejo de estos casos, ya que complican gravemente este estado y constituyen un problema no solo del momento sino que pueden 
conducir a la muerte o dejar secuelas muchas veces de carácter invalidante. Entre éstas se pueden mencionar: edemas dolorosos crónicos, úlceras de la pierna, claudicación funcional, procesos erisipelatosos a repetición, etc. El conjunto de tales fenómenos se ina agrupado bajo la denominación genérica de "síndromes postflebiticos". Estas pacientes pueden, en situaciones desfavorables, nepetir en un nuevo embarazo los trastornos de su coagulación intravascular, aumentando cada vez más la gravedad del problema.

Tratamiento.-Ante todo debe ser profiláctico, iniciado desde la consulta prenatal: un estudio clínico compieto haciendo hincapié sobre el aparato cardiovascular es muy importante y en caso necesario debe solicitarse la cooperación del cardiólogo. El cuidado del sistema venoso es muy importante, ya que hemos visto cómo el terreno varicoso aumenta la incidencia de trombosis. Deben combatirse los estados de adiposidad, de deficiencias nutritivas y sobre todo los de anemia por su repercusión sobre el factor parietal y sobre la composición bioquímica de la sangre. Es importante también la extirpación de focos sépticos en amígdalas, dientes, etc., o su tratamiento médico en caso de sinusitis, pielitis, colecistitis, etc., porque crean un estado de déficit orgánico general crónico y predisponente.

Una vez diagnosticada la trombosis venosa debe instituírse sin dilación el tratamiento, pues de su oportunidad depende en gran parte el éxito (2).

En principio, y como base fundamental, debe tenerse la noción muy precisa de que es una entidad qua requiere tratamiento médico. Sinembargo algunos casos particuiares requieren de la cirugía como recurso muy valioso. De acuerdo con este concepto cebe advertirse, además, que las drogas esenciales son los anticoaguiantes cuya administración debe ser completa y controlada, porque de lo contrario es mejor no dar nada. Al lado de esta medicación deben estar los analgésicos, los antianémicos, incluyendo entre éstos la transfusión y en ocasiones los vasodilatadores específicos o los bloqueos simpáticos regionales. No debe olvidarse que $\in l$ reposo aumenta la estasis circulatoria y por consiguiente una movilización prejoz y racional debe establecerse con oportunidad. Debe tenerse presente que algunos medicamentos, tales como la penicilina, la aureomicina, la vitamina $\mathrm{K}$, los diuréticos mercuriales, la cortisona y sus similares aumentan la coaguiación del plasma y por consiguiente no son recomendables, y antes bien, están contraindicados. 
Medicación anticoagulante.-Su administración está dirigida a impedir la formación de trombos y principalmente a inhibir la extensión de los ya formados (3). Se dividen en dos grupos: I. Heparínicos: heparina, paritol, treburon y II. Depresores de la protrombina, que comprende: a) las cumarinas: dicumarol, cyclocumarol, marcoumar y tromexan, y b) las indandionas: fenilindandiona y dipaxin.

Por la seguridad en su administración, tolerancia y escasos efectos secundarios recomendamos especialmente la heparina y el tromexan, solos o mejor aún asociados para su administración.

La heparina actúa directamente sobre el plasma y por consiguiente sus efectos se manifiestan en contados minutos. Por la misma razón su eliminación es rápida. Se presenta en dosificaciones de 50 miligramos (5.000 U. I.) por c. c. para administración intravenosa. Su antídoto es el sulfato de protamina.

El tromexan (ester etílico del ácido bis 3,3' -4-oxicumarinil acético) actúa por inhibición de la formación del complejo protrombínico en el hígado. Se presenta conteniendo 300 miligramos por comprimido. Reduce la protrombina a un nivel terapéutico eficaz en 48 a 60 horas. Su antídoto, en caso necesario, es la vitamina $\mathrm{K}$.

Estes dos medicamentos, heparina y tromexan, puedien y deben administrarse no solamente en los casos de trombosis venosas durante el puerperio, sino también en el estado de gravidez. Desafortunadamente las estadísticas en estos últimos casos, entre nosotros, no permiten demostrar gráficamente la bonciad de esta conducta, pero vale la pena mencionar a Barry y olson (4), quienes después de analizar una estadística americana de 135 casos la preconizan, advirtiendo que su control debe ser estricto. De 38 casos tratados con anticoagulantes en el curso del embarazo, 7 hicieron embolía pulmonar sin defunciones, y de las 97 no tratadas con anticoagulantes, 18 tuvieron embolía pulmonar y 15 de ellas murieron.

Thomas Burns (2) recomienda el tratamiento de la tromboembolia durante el embarazo con heparina, y en relación con el dicumarol advierte que durante su administración pueden producirse desprendimientos de placenta y muerte fetal. Sinembargo Davis y Mansell, citados por Barry (4), reportan casos de tratamiento prolongado con esta droga sin lesionar al feto. Por lo demás está demostrado que el tromexan es mucho más manejable que el dicumarol, ya que éste último puede producir grandes 
variaciones en la concentración de protrombina por acumulación be dosis.

Si recordamos que en las trombosis venosas predomina el estado de hipercoagulabilidad plasmática, es preciso decir que una paciente normocoagulable es aquélla que tenga una actividad de protrombina de $100 \%$ y un test de tolerancia a la heparina de 11 minutos aproximadamente. El criterio de normocoagulabilidad no se puede determinar por uno solo de estos datos ni mucho menos servir aisladamente de método para el control terapéutico, ya que hay pacientes que tienen una actividad de protrombina vecina del $100 \%$, y sinembargo un test de tolerancia a la heparina revela cifras de hipercoagulabilidad, por ejemplo, de 4-5 minutos.

En términos generales una buena norma terapéutica a seguir en el curso de la administración de anticoagulantes es mantener el test de tolerancia a la heparina entre 1: y 22 minutos, descendiendo sin riesgos la protrombina hasta un 20 a $30 \%$. La observación estricta de estos dos datos es indispensable, porque pueden suceder varios hechos: 10 Que para lograr un test de heparina de 11 o más minutos, tengamos que llevar la protrombina a niveles peligrosos. 2? Que a pesar de un gran descenso de las cifras de protrombina no se logre llevar el test de heparina a niveles terapéuticos eficientes. 39 Que a pesar de la medicación bien administrada no logremos corregir la hipercoagulabilidad. Esta última eventualidad afortunadamente es muy rara.

Se debe considerar como criterio de curación biológica (5) el momento en que diespués de mantener un buen tiempo de tolerancia a la heparina con cifras bajas de protrombina, ésta última comienza a ascender. En este punto se puede considerar que la paciente está curada. Sinembargo, para evitar recidivas se ha de suministrar una dosis de mantenimiento durante un tiempo más o menos largo con controles de laboratorio más espaciados.

A manera de esquema práctico el tratamiento debe iniciarse con heparina administrada en dosis repetidas (50 a 100 miligramos) cada 4 horas, por vía intravenosa o en goteo continuo y lento durante las primeras 24 horas. En adelante se continúa con dosis de tremexan repetidas cada 6 u 8 horas, 900 a 1.200 miligramos en las 24 horas. Los controles de laboratorio nos darán en adelante la pauta terapéutica a seguir.

Medicación analgésica.-Debe incluir principalmente los saIicilatos que reducen la coagulación sangu'nea y alivian el dolor. En casos especiales, principalmente cuando hay espasmo arterial 
reflejo que compromete gravemente la irrigación de los miembros, están indicados la papaverina y los bloqueos del simpático, que a ia vez que combaten el espasmo calman el dolor. También se recomienda la aplicación local de compresas calientes con solución de sulfato de magnesio, evitando hacer masajes sobre la región enferma.

Miedicación vasodilatadora.-Está integrada por los medicamentos específicos y dentro de éstos principalmente ia papaverina y sus similares y por los bloqueos regionales del simpático, bien sean anestésicos o por inyección intra-arterial de procaína, priscol e hydergyna. En algunos casos da magníficos resultados la anestesia peridural después de la administración de la terapéuiica anticoagulante, porque produce analgesia completa y vasodilatación arterial.

Ultimamente se han obtenido buenos resultados en el tratamiento de estos estados con la butazolidina, pero su farmacodinamia aún no está bien establecida en relación con la coagulación. El ideal es asociarla con los anticoagulantes, ya que actuando sobre el aspecto inflamatorio del problema favorece su regresión, alivia a las pacientes y acorta el tiempo de tratamiento. Es bueno advertir que en los casos de trombosis venosa en el curso de la gestación, la administración de anticoagulantes debe reducirse en los días de preparto, no por temor a las hemorragias del alumbramiento, que como es obvio obedecen a otros mecanismos, sino por ia posibilidad de que sea necesario practicar una intervención quirúrgica, tal como la cesárea, para resolver una distocia.

En relación con la movilización de las pacientes afectadas por la trombosis venosa, debemos recalcar sobre la importancia de combatir la estásis circulatoria. De ahí que el levantarlas precozmente esté perfectamente indicado y ello debe hacerse cuando los fenómenos inflamatorios y subjetivos hayan desaparecido, lo cual ocurre más o menos en 5 a 7 días, cuando se han seguido las normas anteriormente expuestas.

Una recomendación importante es la aplicación de un vendaje o en su defecto una media elástica en el miembro enfermo, porque de esta manera provocamos alivio al dolor, reducimos el edema y evitamos las posibles secuelas. El vendaje debe aconsejarse desde la iniciación del tratamiento, y su uso debe prolongarse durante un tiempo más o menos largo.

Finalmente, algunos casos especiales requieren de la cirugía. Tales serían: el tromboembolismo pulmonar a repetición, el fra- 
caso en la medicación anticoagulante bien orientada, las grandes trombosis que comprometan seriamente la anatomía del miembro enfermo. Dentro de estas eventualidades es menester contar con las siguientes posibilidades quirúrgicas: flebotomía y trombectomía, seguida de fleborrafia. Sección venosa, trombectomía y ligajura. Ligadura venosa simple. Flebectomía con trombectomía por aspiración de los cabos venosos central y periférico seguida de ligadura venosa (6).

Un estudio estadístico realizado por nosotros en nuestro medio nos permitirá posteriormente reafirmar algunos conceptos emiticlos en este resumen y algunos casos especiales ilustrarán mejor nuestras ideas en relación con este problema.

\section{BIBLIOGRAFIA}

1. SALDARRIAGA ALBERTO.-“Tromboflebitis y Flebotrombosis". Revista UNIDIA. Volumen II. Número 7. Abril $/ 54$.

2. THOMAS BURNS.- "Thromboembolic disease in Obstetrics and Ginecology'. Am. J. of Obs. and Gy. Vol. 71, número 2. /56.

3. JOHN H. OLWIN.- "Principios de la terapéutica anticoagulante y su aplicación". Copia mimeográfica de la Biblioteca de las Fuerzes de Policía.

4. BARRY and MERLIN J. OLSON.-"Antepartum thombophlebitis". Am. of Obs. and G. Volumen 72, número 4. /56.

5. SOLANO ARMANDO.-“Medicación anticoagulante". Revista UNIDIA. Volumen I. Julio. /55.

6. OSEGUERA DANIEL. M. S. V. C. Caracas. Revista "El Médico". 1958. Página 10. 